\title{
Immunological and redox biomarkers of neutrophils function in HIVIAIDS disease
}

\author{
Serge Blaise Emaleu* and Kondala Atkuri
}

Address: Rabindra Tirouvanziam, Bita Sahaf, Andrew Zolopa, Leonore Herzenberg, Leonard Herzenberg Stanford University School of Medicine, California 95305, USA

* Corresponding author

from Frontiers of Retrovirology: Complex retroviruses, retroelements and their hosts

Montpellier, France. 21-23 September 2009

Published: 24 September 2009

Retrovirology 2009, 6(Suppl 2):P35 doi:10.1186/1742-4690-6-S2-P35

This abstract is available from: http://www.retrovirology.com/content/6/S2/P35

(c) 2009 Emaleu and Atkuri; licensee BioMed Central Ltd.

Previous investigators have shown that HIV/AIDS disease is associated with persistent bacterial and fungal infection which would otherwise be cleared by a functional innate immune system. These observations support the idea that although HIV virus infects only CD4 T cells, the disease leads to an impairment of both innate and adaptive immune system.

Our study hypothesizes that HIV disease and specific treatments thereof (e.g., protease inhibitors) modulate the activity of leukocyte subsets that are not conventional targets for HIV infection, most notably neutrophils. Furthermore, leukocyte (neutrophil) redox and/or functional imbalance underlies important events in HIV disease, including appearance of opportunistic infections.

This study of Neutrophils activation of HIV infected patient helped revisit current immunometabolic concepts that fail to explain the whole spectrum of symptoms in HIV disease in vivo. It has revealed new knowledge about how redox changes within HIV infection. In doing so, achieved better understanding of innate immune subsets, especially neutrophils, in the context of HIV disease. Indeed, neutrophils may be impacted by HIV disease progression (i.e., in their function and not only their numbers), but also, HIV disease progression may in turn be impacted by neutrophil dysfunction (i.e., I the context of failed innate immune response to opportunistic pathogens). Hence, our study delineates simple whole blood biomarkers that will ameliorate the clinical monitoring and prognostic capabilities in HIV disease.
We determined that HIV patients show redox abnormalities (low glutathione, ROS, etc) in neutrophils (and other blood leukocytes), and some functional abnormalities in granulocytes (activation status, phagocytosis, signaling pathways), that associate with disease and treatment history. 